\title{
Le rôle des accords de Schengen dans la construction européenne
}

\section{The Role of the Schengen Agreements in the European Construction}

\section{Charles Elsen}

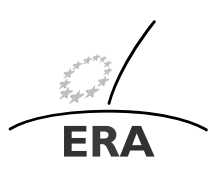

EUROPÄISCHE RECHTSAKADEMIE ACADEMY OF EUROPEAN LAW ACADEMIE DE DROIT EUROPEEN ACCADEMIA DI DIRITTO EUROPEO TRIER - TREVES - TREVIRI

Résumé Les accords de Schengen sont nés à partir d'une coopération intergouvernementale entre cinq États membres. Leur développement a contrasté avec les efforts peu fructueux menés parallèlement au sein des Communautés Européennes pour réaliser la libre circulation des personnes. L'intégration de l'acquis de Schengen dans l'Union européenne a été réalisée par le Traité d'Amsterdam. Les accords de Schengen sont un exemple intéressant des opportunités qu'offre la «coopération renforcée ».

Mots clés Accords de Schengen - Espace de liberté, de sécurité et de justice - Traité d'Amsterdam · Coopération renforcée

Abstract The Schengen Agreements initially result from an intergovernmental cooperation among five Member States. Their development is contrasting with the rather unsuccessful efforts made in parallel in the framework of the European Communities. The integration of the Schengen acquis into the European Union is the result of the Amsterdam Treaty. The Schengen Agreements are an interesting example of the possibilities offered by "enhanced cooperation".

Keywords Schengen Agreements - Area of freedom security and justice · Amsterdam Treaty $\cdot$ Enhanced cooperation 
«Coopération renforcée avant la lettre, l'accord de Schengen et tout le dispositif juridique qui en découle (accords d'adhésion et droit dérivé) constituent un exemple unique de la mise en ouvre par certains États membres d'un objectif communautaire fixé par le traité CE en dehors du cadre institutionnel de ce dernier, avant d'y être "rapatrié" par le traité d'Amsterdam. ${ }^{1}$

\section{Introduction}

A à peine $50 \mathrm{~km}$ de distance, Trèves et Schengen sont toutes les deux situées sur les bords de la Moselle. Le weekend du 12-13 juin 2010 elles ont été le théâtre d'évènements sans rapport direct entre eux, mais méritant d'être mis en relation car relevant de la construction européenne au sens large. Alors que le 12 juin John Toulmin quittait, après 14 ans, ses fonctions de président du Board of Trustees de l'ERA à Trèves, le lendemain fut célébré à Schengen le $25^{\mathrm{e}}$ anniversaire des Accords de Schengen en présence notamment du Président du Parlement Européen, du Président de la Commission Européenne, de nombreux ministres des affaires étrangères et des plus hauts représentants du Grand-Duché de Luxembourg, pays hôte. A cette occasion, le Musée Européen à Schengen a été inauguré.

L'édition de ce liber amicorum me donne l'occasion de souligner les mérites de John Toulmin à la tête du Comité Consultatif de l'ERA qu'il a dirigé avec beaucoup de compétence et de doigté, contribuant ainsi de façon décisive au développement de l'ERA et à l'évolution de ses programmes. Il me donne aussi l'occasion d'une réflexion sur l'histoire des accords de Schengen et les leçons qui peuvent en être tirées. $^{2}$

Trois aspects de la coopération Schengen seront examinés dans cette étude :

- d'abord l'apport respectif des membres fondateurs de Schengen, soit l'Allemagne et la France à travers l'Accord de Saarbrücken et les Pays du Benelux à travers le mémorandum du 12 décembre 1984 qui est à la base de leur collaboration dans ce qui deviendra la coopération Schengen;

- ensuite une réflexion sur les mérites de la coopération renforcée : on en tirera la conclusion que la coopération renforcée, accompagnée du mécanisme de la mise en vigueur, permet d'obtenir un haut degré de convergences parmi les États membres qui participent;

- enfin une réflexion sur l'intégration de Schengen dans l'Union européenne : il en résulte que sans le degré de convergence réalisé grâce aux accords de Schengen, l'espace de liberté, de sécurité et de justice n' aurait pas pu être réalisé ou ne l'aurait été que beaucoup plus tard et de façon moins complète.

\footnotetext{
${ }^{1}$ de Lobkowicz [2], p. 25.

${ }^{2}$ L'ERA consacre régulièrement des séminaires au développement et au fonctionnement de l'Espace Schengen, la proximité géographique entre Trèves et Schengen permet d'ailleurs de combiner ces séminaires avec des visites sur place.
} 


\section{La genèse des accords de Schengen $^{3}$}

\subsection{Généralités}

Lorsque le 13 juillet 1984, Roland Dumas, ministre français des Affaires étrangères et Waldemar Schreckenberger, Secrétaire d'État au Bundeskanzleramt, apposent leur signature sous ce qu'il est convenu d'appeler l'Accord de Saarbrücken, ${ }^{4}$ ils ne se doutent certainement pas qu'ils posent la première pierre d'un édifice que nous appellerons quinze ans plus tard - dans le traité d'Amsterdam - l'espace de liberté, de sécurité et de justice. Nous sommes au lendemain du Sommet de Fontainebleau (25/26 juin 1984) qui vient, dans ses conclusions demander au Conseil de «parvenir ... avant la fin du premier semestre 1985... à la suppression de toutes les formalités de police et de douane aux frontières intercommunautaires pour la circulation des personnes $\gg .5$

Ce geste semble devoir être interprété dans un double sens : d'une part comme symbolisant, de la part du couple franco-allemand, qu'un assouplissement du contrôle aux frontières est possible, et d'autre part, que cette mesure poursuit également un but économique : éviter que l'échange de marchandises (par camion) soit ralenti et par voie de conséquence renchéri par les files d'attente interminables à la frontière commune. L'Accord de Saarbrücken est peu connu : il est donc utile d'en rappeler l'histoire et le contenu.

\subsection{L'Accord de Saarbrücken}

On peut s'étonner que quelques jours après le Sommet de Fontainebleau, la France et l'Allemagne signent un accord qui constitue un essai de mise en œuvre pratique des mesures annoncées dans le domaine de la libre circulation des personnes. L'Accord de Saarbrücken ne semble toutefois pas être un premier résultat des avancées réalisées au Sommet de Fontainebleau. Sa date (moins de trois semaines après ce Sommet) et le fait que dans les considérants aucune référence ne soit faite à ce Sommet amènent à la conclusion que les deux instruments sont indépendants l'un de l'autre.

C'est apparemment le chancelier Kohl qui prend l'initiative d'informer les États Benelux de l'initiative franco-allemande, en les invitant à s'y joindre. La Belgique, les Pays-Bas et le Luxembourg ont conclu, presque 25 ans plus tôt, une convention concernant le transfert du contrôle des personnes vers les frontières extérieures du territoire du Benelux. ${ }^{6}$ Le Benelux répond favorablement à l'invitation et transmet aux Français et Allemands un Mémorandum daté du 12.12.1984. ${ }^{7}$ Désormais la voie est libre pour une coopération à cinq.

\footnotetext{
${ }^{3}$ L'expression « accords de Schengen » vise à la fois l'Accord du 14 juin 1985 relatif à la suppression graduelle des contrôles au frontières communes et la Convention d'application de l'accord de Schengen du 14 juin 1985, (CAAS) signée le 19 juin 1990. OJL 239 du 22.9.2000.

${ }^{4}$ Accord entre le Gouvernement de la République Française et le Gouvernement de la République fédérale d'Allemagne relatif à la suppression graduelle des contrôles à la frontière franco-allemande (13 juillet 1984 J.O. de la République Française 3.8.1984, p. 485-489).

${ }^{5}$ Conseil européen de Fontainebleau, Conclusions de la Présidence doc SN 1446/1/84.

${ }^{6}$ Convention du 11.4.1960.

${ }^{7}$ Non publié.
} 
L'Accord de Saarbrücken, comme d'ailleurs le Mémorandum des pays du Benelux et encore l'Accord de Schengen de 1985 qui est le résultat des premières négociations à cinq, ont beaucoup de points communs. ${ }^{8}$ Le principal consiste à prendre des mesures immédiates concernant les contrôles des ressortissants CEE aux frontières intérieures : le contrôle systématique (prévu mais impraticable de fait vu le flux du trafic) est remplacé par un contrôle par sondages ; les formalités de contrôle en ce qui concerne les ressortissants CEE sont remplacées par une simple surveillance visuelle des véhicules (disque vert à apposer sur le pare-brise du véhicule).

Par ailleurs, après réalisation des mesures immédiates, un programme de travail est annoncé pour le futur.

L'Accord de Saarbrücken prévoit un train de mesures à réaliser (ou à étudier) avant octobre 1984, date du prochain Sommet franco-allemand (deuxième étape) et d'autres mesures à prendre avant le 31 décembre 1986. Les auteurs du mémorandum Benelux, plus prudents, parleront de mesures à plus long terme.

Mais cette prudence s'explique probablement par le fait que les objectifs, du côté du Benelux, sont plus ambitieux.

Ainsi le Benelux préconise, pour la phase 2, la définition et mise en œuvre progressive d'une politique commune concernant l'entrée, la circulation et l'éloignement des étrangers; le document franco-allemand est muet sur ce point. Ainsi encore, le Mémorandum du Benelux énumère l'harmonisation des politiques de visas et des règles de délivrance et propose la création d'un visa valable pour le court séjour pour l'ensemble du territoire des pays contractants, alors que l'Accord de Saarbrücken se borne à annoncer la préparation de l'harmonisation des règles de délivrance des visas exigés par chacune des deux parties.

Le Mémorandum Benelux préconise de façon claire le déplacement du contrôle des personnes des frontières communes aux frontières extérieures alors que le texte franco-allemand parle de la recherche des moyens de transférer aux frontières externes des deux États les contrôles effectués à la frontière interne (article 8). Cet article paraît en contradiction avec l'article 15 al. $1^{\mathrm{er}}$ qui préconise la généralisation des contrôles groupés aux frontières intérieures (la suppression des contrôles n'est donc pas visée) et avec l'art. 15 al. 2 qui cite l'harmonisation des législations dans certains domaines (stupéfiants, armes) comme préalable à la suppression de tout contrôle pour les ressortissants CEE, d'où il semble résulter à contrario que le contrôle des ressortissants des pays tiers échapperait aux mesures de libéralisation prévues.

Deux considérants du Mémorandum Benelux méritent par ailleurs d'être soulignés : d'une part, la référence à la déclaration du Conseil européen de Fontainebleau ${ }^{9}$ et, d'autre part, la référence au fait que le Benelux dispose d'une longue expérience dans ce domaine, le contrôle aux frontières intérieures ayant été aboli grâce à l'harmonisation des législations et en partie grâce au déplacement des contrôles à l'intérieur des trois pays.

Enfin, notons la volonté des pays du Benelux de ne pas fermer la porte à d'autres pays membres de la CE qui souhaiteraient s'y associer : ici parait le souci de ne

\footnotetext{
${ }^{8}$ Cette étude ne porte que sur les aspects concernant la libre circulation des personnes à l'exclusion d'autres questions et notamment de la circulation des marchandises et des questions relatives au transport.

${ }^{9}$ Voir point 2.1.
} 
pas agir contre les intérêts de la Communauté européenne : plus tard on parlera du laboratoire d'essais Schengen. ${ }^{10}$

\subsection{Le résultat de la coopération à cinq : les accords de Schengen}

Moins de douze mois après le Sommet de Fontainebleau et l'Accord de Saarbrücken, six mois après le Mémorandum Benelux, les cinq États fondateurs signent à Schengen l'Accord relatif à la suppression graduelle des contrôles aux frontières communes.

Le texte s'inspire à la fois de l'Accord de Saarbrücken et du Mémorandum Benelux, les considérants font expressément référence aux deux instruments, ainsi qu'à la déclaration du Conseil européen de Fontainebleau. Il maintient la distinction entre mesures réalisables immédiatement ou à bref délai d'un côté, et mesures applicables à long terme (art. 17); il fixe la date du $1^{\text {er }}$ janvier 1990 pour la mise en œuvre de cette deuxième série de mesures. ${ }^{11}$

Comme on le sait, et comme il sera développé ci-dessous, la coopération Schengen ne s'est pas limitée aux cinq États qui ont négocié les Accords de 1985 et 1990 ; actuellement (situation au 31.12.2010) 31 États, dont les 27 États membres de l'Union européenne ainsi que l'Islande, la Norvège, la Suisse et le Liechtenstein participent aux discussions concernant le développement de l'acquis de Schengen; l'espace Schengen, c.à.d. l'espace sans contrôle aux frontières intérieures, fonctionne entre 25 États. $^{12}$

2.4 Réflexion sur le rôle respectif des « grands » et des «petits » États

L'histoire de Schengen apparaît ainsi comme un bel exemple de la façon dont les États membres de l'Union européenne, grands ou petits, peuvent contribuer à la réalisation d'une idée : le couple franco-allemand, comme si souvent dans l'histoire européenne, a joué un rôle de pionnier; les trois pays du Benelux, grâce à leur expérience d'un territoire sans contrôle aux frontières communes, grâce aussi à leur foi dans l'idéal européen, ont pu enrichir cette idée initiale. Avec l'histoire de Schengen se vérifie le constat de Jacques Delors : «On ne comprend rien à la construction européenne si on ne tient pas compte du rôle joué par chaque pays, du plus petit jusqu'au plus grand $\gg .{ }^{13}$

\footnotetext{
${ }^{10}$ Fischbach [8], p. 1.

${ }^{11}$ En fait, et notamment en raison d'évènements historiques (chute du mur de Berlin) ce délai n'a pas pu être respecté : la CAAS ne sera signée qu'en juin 1990 et l'entrée en vigueur des accords, elle aussi retardée, n'interviendra que le 26.3.1995.

${ }^{12}$ Actuellement (situation au 31.12.2010) le contrôle aux frontières intérieures subsiste pour l'Irlande, le Royaume-Uni, Chypre, la Bulgarie, la Roumanie et le Liechtenstein.

${ }^{13}$ Delors [3], p. 220.
} 


\section{Les mérites de la coopération renforcée à l'image des accords de Schengen}

\subsection{Pro et contra la coopération renforcée}

On connaît les réflexions sur les avantages et les inconvénients de la coopération renforcée. Pour les uns, les inconvénients l'emportent : la coopération renforcée détruit l'unité de la règle européenne; elle mène à un morcellement du droit européen. Pour les autres, la coopération renforcée est une voie de progrès, surtout dans les matières régies par la règle de l'unanimité : lorsque la règle de l'unanimité empêche tout consensus à un niveau acceptable, il est préférable de permettre à certains États - à défaut de tous - de progresser. C'est cette thèse qui l'a finalement emporté dans les traités européens et successivement le traité d'Amsterdam, ${ }^{14}$ le traité de Nice ${ }^{15}$ et le traité de Lisbonne ${ }^{16}$ sont venus préciser les conditions de la coopération renforcée dans le cadre de l'UE.

Les accords de Schengen ne sont pas une coopération renforcée stricto sensu : ils n'ont pas été négociés dans le cadre des Communautés européennes, mais en dehors. Au début des travaux de Schengen, la possibilité d'une coopération renforcée, au sens des traités européens, n'existait pas; la coopération dans le domaine de la justice et des affaires intérieures ne sera mise sur les rails que par le traité de Maastricht, postérieur aux accords de Schengen.

Sur la route menant vers l'espace de liberté, de sécurité et de justice, telle que défini par le traité d'Amsterdam et explicité par les Conclusions du Sommet de Tampere ${ }^{17}$ la coopération Schengen fait figure de précurseur. On examinera successivement le contenu (3.2), les structures (3.3), les procédures de mise en vigueur (3.4), le développement (3.5) et les faiblesses (3.6) de la coopération Schengen.

\subsection{Le contenu des accords de Schengen}

Le contenu des accords de Schengen se présente comme un ensemble cohérent et bien structuré. Partant d'une idée simple - la suppression du contrôle aux frontières intérieures - on imagine un système complet de mesures devant rendre possible cette mesure qui donnera enfin son plein sens à la notion de libre circulation des personnes, inscrite déjà dans le traité de Rome du 25 mars $1957 .{ }^{18}$ Cette mesure n'est pas facile à réaliser, car elle a des implications dans de nombreux domaines. Elle exige des mesures de contrôle aux frontières extérieures d'un niveau suffisant : désormais, ce contrôle remplacera le contrôle à la frontière intérieure. Elle exige une politique des visas commune, une coopération policière et judiciaire renforcées. Si en matière de coopération judiciaire les États Schengen peuvent s'appuyer sur les conventions performantes du Conseil de l'Europe dans le domaine de l'extradition et de l'entraide

\footnotetext{
${ }^{14}$ Signé le 2.10.1997 et entré en vigueur le 1.5.1999 (JO C 340 du 10.11.1997).

${ }^{15}$ Signé le 26.2.2001 et entré en vigueur le 1.2.2003 (JO C 80 du 10.3.2001).

${ }^{16}$ Signé le 13.12.2007 et entré en vigueur le 1.12.2009 (JO C 306 du 17.12.2007).

${ }^{17}$ Voir Conseil européen de Tampere 15-16.10.1999. Conclusions de la Présidence SN 200/99.

${ }^{18}$ Traité CEE, article 3c.
} 
judiciaire, il n'en est pas de même en matière de coopération policière où la coopération sur le plan européen se limite essentiellement à des accords bilatéraux relatifs à la coopération locale dans le périmètre de la frontière commune : se basant sur des modèles tirés de la coopération Benelux - qui permet le droit de suite ${ }^{19}$ - ou en innovant par l'utilisation de techniques modernes - comme la création du Système Informatique Schengen (SIS) ${ }^{20}$-, les accords de Schengen définissent un nouveau modèle de coopération policière en Europe. Enfin, dans certaines matières sensibles, comme les armes prohibées ou les stupéfiants - qui rendaient nécessaire un contrôle à la frontière - un rapprochement des législations a pu être réalisé, et, pour assurer le fonctionnement du SIS, une mise à niveau des législations sur la protection des données a été incorporée.

\subsection{Les structures de Schengen}

Dans la phase de négociation (1985-1990) comme dans la phase de ratification (1990-1995) et par la suite après l'entrée en vigueur (de 1995 au 1.5.1999, date de l'intégration dans l'Union européenne), le système Schengen fonctionnait sur la base d'une structure de type pyramidal. Des groupes d'experts préparaient les textes, élaboraient les projets de décision et rédigeaient les instructions. Leur travail remontait à un groupe de type groupe directeur qui préparait le dossier pour les hauts fonctionnaires - essentiellement des Ministères des Affaires étrangères - regroupés dans un groupe appelé "Groupe Central" qui soumettait les projets de décisions au Comité Exécutif, composé de membres des Gouvernements des États participants. ${ }^{21}$

Le traité de Maastricht s'inspirera très clairement de cette structure, le Comité de l'Article K4 prévu dans le traité pouvant être considéré comme le successeur du Groupe Central de la structure Schengen.

Les groupes Schengen ont dès le début profité de l'hospitalité du Secrétariat général du Benelux qui mit à leur disposition un petit secrétariat. Les autres États participaient aux travaux du secrétariat en détachant quelques fonctionnaires nationaux.

Une petite équipe d'interprètes/traducteurs (qui augmentait au fur et à mesure de l'arrivée de nouveaux États membres) complétait ce secrétariat qui réussissait - avec des moyens limités - à accomplir un travail remarquable. ${ }^{22}$

\subsection{La procédure de mise en vigueur}

Il convient de signaler une particularité concernant le moment où les accords de Schengen deviennent applicables dans les États qui y adhèrent. L'acte final de la CAAS contient la déclaration suivante relative à l'article $139:$ : la Convention ne sera

\footnotetext{
${ }^{19}$ Convention Benelux précitée (voir note 6) article 27.

${ }^{20}$ CAAS Titre IV articles 92 à 119.

${ }^{21}$ Pour le détail voir Elsen [5], p. 19 à 26.

${ }^{22}$ Conformément à l'article 7 du Protocole intégrant l'acquis de Schengen dans le cadre de l'Union européenne, le secrétariat de Schengen a été intégré au Secrétariat Général du Conseil. Au moment de l'intégration, le secrétariat Schengen comprenait environ 60 personnes, dont 40 interprètes/traducteurs.
} 
mise en vigueur que lorsque les conditions préalables à l'application de la Convention seront remplies et que les contrôles aux frontières extérieures seront effectifs ».

Une décision du Comité Exécutif du 16 septembre 1998 concernant la création d'une commission permanente d'évaluation et d'application de Schengen organise de façon détaillée les contrôles à effectuer avant la mise en vigueur de la Convention. ${ }^{23}$

\subsection{Le développement « géographique » de l'espace Schengen}

Le développement de Schengen a été remarquable. Il faut dire que les auteurs de Schengen avaient largement favorisé cet élargissement en prévoyant que tout État membre des Communautés Européennes pouvait devenir partie à la Convention (art. 140). Par contre, les États candidats devaient accepter le texte sans pouvoir rouvrir une discussion sur le fond. Ainsi le Portugal n'a-t-il pas pu maintenir intégralement, en matière d'extradition et d'entraide judiciaire, sa position concernant les peines privatives de liberté à perpétuité. ${ }^{24}$

Il en est de même pour la Grèce qui, par contre, a réussi à négocier une déclaration commune relative au Mont Athos dans laquelle les Parties contractantes déclarent qu'elles veilleront à tenir compte du statut spécial accordé au Mont Athos dans l'application et l'élaboration ultérieure de l'Accord de 1985 et de la Convention de $1990 .^{25}$

Entre le 19.6.1990 (signature de la CAAS) et le 26.3.1995 (entrée en vigueur des accords de Schengen), l'Italie (1990), l'Espagne et le Portugal (1991), la Grèce (1992) signeront des actes d'adhésion. Entre le 26.3.1995 et le 1.5.1999 (date de l'intégration de l'acquis Schengen dans l'Union européenne), l'Autriche (1995), le Danemark, la Finlande et la Suède (1996) en feront de même, ainsi que l'Islande et la Norvège (1996) qui forment avec eux l'Union Nordique des passeports.

Ainsi, au moment de l'intégration de l'acquis de Schengen dans l'Union européenne en 1999, la coopération Schengen comprend 13 des 15 membres formant alors l'Union européenne. «L'une des leçons des accords de Schengen conclus à l'origine par seulement cinq États membres est d'avoir eu une capacité d'attraction telle que la plupart des États membres y ont finalement adhéré ».26,27

\subsection{Les faiblesses du système Schengen}

Malgré ses succès qui se manifestaient d'un côté, comme on vient de le voir, par des adhésions nombreuses de nouveaux États, et de l'autre côté, par un fonctionnement

\footnotetext{
${ }^{23} \mathrm{SCH} / \mathrm{Com}$-ex (98) 26 déf. Cette décision fait partie de l'acquis de Schengen repris par l'Union européenne.

${ }^{24}$ Accord d'adhésion de la République Portugaise aux accords de Schengen du 25.6.1991 (art. 5 et 6).

${ }^{25}$ Accord d'adhésion de la République Hellénique aux accords de Schengen du 6.11 .1992 (art. 4 et 5) et Acte Final - Déclaration relative au Mont Athos.

${ }^{26}$ de Lobkowicz [2], p. 197.

${ }^{27}$ Depuis l'intégration de l'acquis de Schengen dans le cadre de l'Union européenne, l'article 8 du Protocole Schengen s'applique; il stipule que les États candidats à l'adhésion doivent intégralement accepter l'acquis de Schengen. Dès lors, grace aux adhésions de 2004 et 2007, 12 nouveaux États membres seront liés par les accords de Schengen de même que la Suisse (depuis 2004) et le Liechtenstein (depuis 2008).
} 
somme tout peu problématique des dispositions de la CAAS - l'ouverture des frontières n'avait pas eu comme conséquence une augmentation massive de la criminalité ou de l'immigration illégale, et le fonctionnement du SIS donnait pleine satisfaction - des problèmes institutionnels majeurs se profilaient à l'horizon. Les adversaires de Schengen étaient nombreux et les critiques contre les accords de Schengen se faisaient entendre. Parmi celles-ci le Parlement européen qui aurait voulu être associé aux travaux de Schengen, ce qui était juridiquement impossible. ${ }^{28}$

La Commission, quant à elle, eut une position plus nuancée. Il est vrai qu'elle avait échoué dans son initiative pour alléger le contrôle des ressortissants communautaires aux frontières intérieures. ${ }^{29}$ Par ailleurs, dès le début un rôle d'observateur lui fut reconnu dans différentes enceintes Schengen. Enfin, elle devait être rassurée par le fait que Schengen se présentait comme un laboratoire d'essai, et non comme un concurrent à l'activité menée au sein des Communautés Européennes. L'article 134 CAAS stipule en effet que les dispositions de la CAAS ne sont applicables que dans la mesure où elles sont compatibles avec le droit communautaire. En d'autres termes, une nouvelle disposition communautaire remplace la disposition afférente de Schengen. ${ }^{30}$

Mais les critiques parvenaient aussi des États membres, en particulier du Conseil d'Etat néerlandais. Il était reproché aux accords de Schengen de ne pas avoir de légitimité démocratique (absence d'intervention des parlements nationaux, incompétence du Parlement européen dans le processus d'élaboration du droit dérivé) ; il était reproché également que le contrôle juridictionnel faisait défaut. On peut ajouter d'autres lacunes et faiblesses : pour des questions d'ordre budgétaire il n'avait pas été possible d'assurer un régime linguistique approprié pour le cinq pays nordiques qui avaient adhéré à Schengen en 1996; seul l'anglais a été introduit comme langue de travail supplémentaire. Enfin, on notera qu'aucun système de publication des décisions du Comité Exécutif de Schengen n'était prévu : il n'existait aucune publication officielle du Secrétariat Schengen ni de publication équivalente sur le plan national. Les réunions du Comité exécutif étaient tout au plus suivies de communiqués de presse assez laconiques.

De tout cela on peut déduire que le moment était venu pour des changements d'envergure : la négociation du traité d'Amsterdam allait fournir l'occasion pour le "big bang", c.à.d. l'intégration de l'acquis de Schengen dans l'Union européenne par le biais du Protocole intégrant l'acquis de Schengen dans le cadre de l'Union européenne, annexé au Traité d'Amsterdam. ${ }^{31}$

\footnotetext{
${ }^{28}$ Elsen [6], p. 11 et 12.

${ }^{29}$ de Lobkowicz [2], p. 24.

${ }^{30}$ Fischbach [8], p. 4.

${ }^{31}$ Le Protocole «Schengen », annexé au Traité d'Amsterdam définit les modalités de cette intégration. Une annexe à ce protocole précise ce qu'il faut comprendre sous la notion de « acquis de Schengen » (décision du Conseil du 20.5.1999. 1999/435/CE).
} 


\section{L'intégration des accords de Schengen dans l'Union européenne}

\subsection{Généralités}

On a présenté souvent la coopération dans le cadre de Schengen comme un laboratoire d'essai ${ }^{32}$ pour des décisions à venir dans le cadre de l'UE. J'ai plutôt tendance à présenter Schengen comme une voie parallèle aux travaux menés dans l'UE avec la particularité - peu mathématique, je le concède - qu'en l'espèce les deux parallèles vont se rencontrer. Le Protocole intégrant l'acquis de Schengen dans le cadre de l'Union européenne met fin à la vie indépendante de l'expérience Schengen dont la substance sera intégrée dans l'Union européenne moyennant quelques aménagements. On analysera successivement dans ce chapitre les activités des Communautés, puis de l'Union européenne autour des thèmes constituant la coopération Schengen de 1985 à 1995 (4.2), les différentes possibilités de rapprochement ou d'intégration des accords de Schengen dans l'Union européenne (4.3), les difficultés de cette intégration (4.4). On posera la question de savoir si l'activité Schengen, en parallèle des activités des instances européennes était un frein ou un accélérateur par rapport à la création de l'espace de liberté, de sécurité et de justice, notion qui apparaîtra pour la première fois dans le Traité d'Amsterdam (4.5). ${ }^{33}$ Le chapitre sera complété par quelques réflexions sur la coopération renforcée (4.6).

4.2 Les activités dans le domaine de la libre circulation des personnes au sein des Communautés / de l'Union européenne

Nous avons vu que le sommet de Fontainebleau coïncidait dans le temps avec l'initiative franco-allemande aboutissant à la signature de l'accord de Saarbrücken. ${ }^{34}$ En fait, la libre circulation des personnes est inscrite dans les Traités européens depuis le Traité de Rome de 1957 (art. 3c) mais elle est restée confinée longtemps à deux aspects : la libre circulation des travailleurs et le droit d'établissement. ${ }^{35}$

Le Conseil européen de Fontainebleau adoptera des orientations nouvelles concernant la relance de la coopération européenne. Le chapitre 6 des conclusions, consacré à l'Europe des Citoyens, mettra en place un comité ad-hoc pour préparer et coordonner les différentes actions. ${ }^{36,37}$ Dans le domaine plus précis de la libre circulation des personnes, il demande - avec comme date limite la fin du $1^{\text {er }}$ semestre 1985 - la «suppression de toutes les formalités de police et de douane aux frontières intercommunautaires pour la circulation des personnes ». 38

\footnotetext{
${ }^{32}$ de Lobkowicz [2], p. 114 voir aussi note 10.

${ }^{33}$ L'article $2,3^{\mathrm{e}}$ tiret du TUE enumère parmi les objectifs de 1'Union « de maintenir et de développer l'Union en tant qu'espace de liberté, de sécurité et de justice au sein duquel est assurée la libre circulation des personnes ...».

${ }^{34}$ Voir point 2.2 .

${ }^{35}$ Fally [7], tome 1 p. $211-212$.

${ }^{36} \mathrm{Ce}$ comité est connu sous le nom de comité Adonnino du nom de son président. Il rendra deux rapports qui seront soumis aux Conseils européens de Bruxelles (29-30.3.1985) et Milan (28-29.6.1985). On notera une nouvelle fois la concordance dans le temps avec l'accord de Schengen signé le 14.6.1985.

${ }^{37}$ Sur cette question voir Fally V. p. 340 \& suivantes.

${ }^{38}$ Voir point 2.1. et note (5).
} 
Cet objectif, très ambitieux, ne pourra pas être tenu. En fait, le problème du contrôle des personnes aux frontières intérieures sera abordé de façon claire dans la première modification que le traité de Rome subira par l'adoption de l'Acte Unique Européen (février 1986). ${ }^{39}$ Désormais la question de la libre circulation des personnes - sous son aspect général d'abolition du contrôle aux frontières intérieures - fera partie des travaux devant aboutir à l'établissement d'un grand marché intérieur pour lequel la date de réalisation est fixée par le nouveau traité au 31.12.1992. Mais l'abolition du contrôle des personnes aux frontières soulève une série de problèmes de la compétence des ministres de l'intérieur et de la justice : problèmes liés à l'immigration et à l'asile, problèmes liés à la lutte contre la criminalité y compris des formes particulièrement graves comme le terrorisme ou le trafic de drogues. De fait le programme auquel l'Europe est confrontée s'apparente à celui esquissé dans l'accord de Schengen de 1985. Or, à ce stade, il n'existe pas de structures de travail appropriées au sein des institutions européennes. ${ }^{40}$

Un «groupe ad-hoc immigration » sera mis en place en octobre 1986 à l'initiative de la présidence britannique. ${ }^{41}$ Ce groupe préparera notamment la convention de Dublin. ${ }^{42}$ On se rendra compte toutefois très rapidement que les travaux vont traîner en longueur et il apparaît nécessaire d'établir un plan d'ensemble pour couvrir tous les aspects liés à l'abolition du contrôle des personnes aux frontières. Le Conseil européen de Rhodes instaurera un groupe de coordinateurs (décembre 1988); ceux-ci élaboreront un document de synthèse dit «document de Palma » qui sera adopté par le Conseil européen de Madrid en juin 1989. Le document de Palma établit un programme d'ensemble pour les années à venir en vue d'atteindre l'objectif de la libre circulation des personnes à partir du 1.1.1993. Chaque Conseil européen était saisi d'un rapport d'étape ; malgré cette structuration des travaux les objectifs ne pourront pas être atteints. ${ }^{43}$

Ainsi, au moment où dans la coopération Schengen, la convention d'application de l'accord de 1985 avait déjà presque été finalisée, les instances européennes n'en sont qu'à l'élaboration d'un plan d'action. De plus, les travaux concrets ne progressent que difficilement : ainsi le dossier sur le contrôle des personnes aux frontières estil abordé dans un premier temps sous son seul aspect «frontières extérieures », réservant à plus tard le problème, plus difficile, de l'abolition du contrôle aux frontières communes; or, après trois ans de négociations, la convention «frontières extérieures » échoue (1.7.1991). ${ }^{44}$

\footnotetext{
${ }^{39}$ de Lobkowicz W. [2], p. 28.

${ }^{40}$ Mis à part la coopération dans la lutte contre la drogue remontant à 1972 et connue sous le nom de groupe Pompidou, et la lutte contre le terrorisme (groupe TREVI) depuis le sommet de Rome de décembre 1975; les deux groupes fonctionnent sur une base intergouvernementale et en dehors des traités.

${ }^{41}$ Pour le détail voir de Lobkowicz. [2], p. 29.

${ }^{42}$ Convention de Dublin sur l'Etat membre responsable de l'examen d'une demande d'asile (J.O. C 254 du 19.8.1997).

${ }^{43}$ Voir paragraphes suivants et note 44 .

${ }^{44}$ De ce jour date le début de l'inexorable logique qui devait conduire à réaliser la libre circulation des personnes par des élargissements successifs de Schengen pour, à terme, en intégrer le résultat dans le Traité d'Amsterdam. de Lobkowicz [2], p. 32.
} 
Plusieurs éléments ont favorisé les travaux dans les enceintes Schengen par rapport aux enceintes communautaires : un plus petit nombre de pays participants, permettant une unité de vues plus grande, une meilleure structuration au niveau des groupes de travail et le fait que dans la coopération Schengen, les dossiers étaient gérés (du moins jusqu'en 1995) par les ministères des affaires étrangères (ou européennes) et non par les ministères techniques tels que l'intérieur ou la justice. Ce n'est qu'avec l'entrée en vigueur du Traité de Maastricht que l'Union européenne sera mise en position d'aborder ces problèmes de façon systématique et coordonnée. ${ }^{45}$

\subsection{Le Traité d'Amsterdam}

A ce stade il convient de jeter un regard sur les structures de la coopération Schengen et celles qui ont été mises en place dans l'Union européenne par le Traité de Maastricht. ${ }^{46}$ On constate que la structure générale depuis les réunions ministérielles jusqu'aux groupes de travail est analogue (avec la seule exception que le COREPER n'a pas d'équivalent dans la structure Schengen); dans le détail nous retrouvons dans l'une comme dans l'autre enceinte les mêmes groupes. Ainsi il existe, d'un côté comme de l'autre, un groupe visa, un groupe asile, un groupe frontières extérieures ou encore, en matière de lutte contre la criminalité, un groupe police et sécurité dans Schengen dont l'équivalent dans la structure de Maastricht est le groupe directeur police-douanes. Il n'y a guère que la coopération judiciaire qui est plus développée dans la structure du TUE, ce qui mènera logiquement, dans le Traité d'Amsterdam, à la création de l'espace de liberté, de sécurité et de justice, alors que la coopération Schengen se développe essentiellement autour des deux pôles sécurité et liberté. ${ }^{47}$

C'est la présidence néerlandaise qui aborda de front le problème de l'intégration de l'acquis de Schengen dans le cadre de la conférence intergouvernementale précédant le Traité d'Amsterdam. Plusieurs scénarii avaient été envisagés pour amener un rapprochement respectivement une intégration des travaux menés dans les deux enceintes. Une première approche visait à réaliser l'intégration par étapes. Une autre alternative consistait à inscrire dans le Traité d'Amsterdam le principe de l'intégration de Schengen, tout en réservant la date de cette intégration à une décision ultérieure du Conseil à prendre à l'unanimité. La troisième alternative - la plus audacieuse consistait à décider que l'intégration de Schengen deviendrait effective au moment de l'entrée en vigueur du Traité d'Amsterdam. C'est cette dernière solution qui fut finalement retenue. Elle a été facilitée par le fait que 13 parmi les 15 États membres de l'Union avaient entretemps rejoint la coopération Schengen. Mais de sérieux problèmes restaient à résoudre. ${ }^{48}$

\footnotetext{
${ }^{45}$ Sur cette question voir Papagianni G. [9], p. 109-113. L'auteur y oppose « the Single European Act : a promise not kept» à « Schengen : the successful laboratoire d'essai ».

${ }^{46}$ Voir Elsen [5], p. 19 à 26.

${ }^{47}$ Fischbach [8], p. 3.

${ }^{48}$ Voir Papagianni [9], p. 35 à 41. L'auteur qualifie l'incorporation de l'acquis de Schengen dans l'UE comme «the return of the prodigal son».
} 
4.4 Les problèmes posés par l'intégration de l'acquis de Schengen dans l'Union européenne

Il faut louer le courage des négociateurs du Traité d'Amsterdam d'avoir choisi l'option la plus radicale et d'avoir trouvé des solutions à quelques problèmes d'une grande complexité. ${ }^{49}$ Quatre questions méritent d'être brièvement analysées.

\subsection{1}

La position du Royaume-Uni et de l'Irlande. Les deux pays qui avaient toujours déclaré ne pas pouvoir participer aux accords de Schengen, ne se sont toutefois pas opposés à l'intégration de Schengen dans l'Union européenne; il y avait néanmoins un prix politique à payer : ces deux pays ne sont pas liés par les accords de Schengen - qui deviennent de ce fait la première coopération renforcée et, ils obtiennent une possibilité d'opt-in, totale ou partielle. ${ }^{50}$

\subsection{2}

La position des États membres de Schengen, mais non membres de l'Union européenne, demandait une solution appropriée. ${ }^{51}$ Nous avons vu ci-dessus ${ }^{52}$ que la Norvège et l'Islande ont rejoint Schengen en 1996, ils feront partie de l'espace Schengen à partir du 15 mars 2001. Il a fallu régler dans le détail leur participation dans la négociation et la prise de décisions concernant le développement de l'acquis de Schengen. La solution retenue consistait à faire participer la Norvège et l'Islande à l'élaboration des instruments juridiques (decision shaping) mais non, pour des raisons institutionnelles, à la prise de décision (decision taking). Un comité mixte, dans lequel les deux pays étaient membres, a été institué à tous les niveaux de l'élaboration des décisions concernant le développement de l'acquis de Schengen. ${ }^{53}$

\subsection{3}

Pour des raisons de sécurité juridique, le Conseil a défini en quoi consistait l'acquis de Schengen et a défini des bases juridiques - dans le premier et dans le troisième pilier - pour le développement de cet acquis.

\footnotetext{
${ }^{49}$ Sur ces problèmes voir l'analyse détaillée dans de Lobkowicz [2], p. 112-120 ou encore Elsen [6], p. 1120.

${ }^{50}$ Voir Traité d'Amsterdam - Protocole intégrant l'acquis de Schengen dans le cadre de l'UE articles 4 et 5 (JO C 340 du 10 novembre 1997). Opt-in du Royaume-Uni : voir décision du 29.5.2000 - opt-in partiel - entrée en vigueur le 1.1.2005. Opt-in partiel de 1'Irlande : décision analogue du 28.2.2002, non encore en vigueur.

${ }^{51}$ Voir Papagianni [9], p. 33 et 34.

${ }^{52}$ Voir chapitre 2.5 .

${ }^{53}$ Voir décision du Conseil 1999/439 CE du 17.5.1999 JO L 176 du 10.7.1999, p. 35-62.
} 


\subsection{4}

Pour le développement du droit dérivé, il est important de déterminer si une mesure constitue, ou non, un développement de l'acquis de Schengen. Selon le cas, le Royaume-Uni et l'Irlande peuvent en être exclus, les États non membres de l'Union (actuellement Norvège, Islande et Suisse) étant liés par cet instrument. La CJCE a eu à trancher plusieurs affaires. ${ }^{54}$

\subsection{La coopération Schengen - frein ou accélérateur?}

On a vu que la coopération Schengen, d'abord conçue comme un laboratoire d'essai des travaux dans l'UE, s'est transformée plutôt en voie parallèle, lorsque, après l'entrée en vigueur du Traité de Maastricht, les structures au sein de la Commission et du Conseil ont été mises en place et que le Conseil des Ministres, dans sa formation JAI (Justice et Affaires Intérieures) s'est mis au travail à partir de 1994. La compétition qui s'est instituée entre ces deux enceintes, pourtant si différentes, a eu des aspects tant positifs que négatifs. Dans la partie négative du bilan nous rangerons les nombreuses enceintes qui faisaient double emploi et menaient à des solutions parfois bizarres (p.ex. en matière d'établissement des listes des pays soumis à l'obligation de visa). Cette situation aurait pu perdurer si, comme par miracle, on n'avait pas réussi à faire converger les deux parallèles constituées par les accords de Schengen d'une part et le Traité de Maastricht d'autre part.

A défaut de cette solution, les travaux de Schengen se seraient probablement arrêtés un jour faute de trouver des réponses aux questions non résolues, de l'absence de contrôle parlementaire et de contrôle juridictionnel et pour des questions administratives et budgétaires. A l'inverse, la coopération Schengen peut, sans hésitation, être qualifiée d'accélérateur de la réalisation de l'espace de liberté, de sécurité et de justice. Sans le laboratoire Schengen, la suppression du contrôle aux frontières intérieures n'aurait pu être réalisée dans un délai raisonnable, aucune des tentatives de la Commission et des actions du Conseil n'ayant abouti dans les délais fixés par l'Acte Unique. $^{55}$

Les avancées réalisées par Schengen dans le domaine de la coopération policière, avec notamment l'introduction du droit de suite, touchant directement aux droits de souveraineté des États, ne paraissaient pas réalisables dans une enceinte européenne plus large. Il en est de même du système informatique Schengen qui a amélioré de façon spectaculaire les moyens de coopération entre les polices de différents États.

Le démarrage de la coopération Schengen à cinq (tous des États membres fondateurs de l'Europe de Schuman et de Monnet) a permis d'établir un socle commun de règles et d'ambitions permettant d'atteindre le but fixé. Deux éléments ont ensuite été déterminants pour maintenir ce haut niveau de convergence :

- d'un côté, le maintien strict de la ligne de conduite selon laquelle rien n'était négociable pour les pays désireux de joindre la coopération Schengen : en d'autres mots le paquet négocié à cinq devait être accepté en bloc par les nouveaux candidats ;

\footnotetext{
${ }^{54}$ Voir par exemple affaire C-482/08 Royaume-Uni c/ Conseil de l'UE, arrêt du 26.10.2010.

${ }^{55}$ Voir notamment point 4.2 et notes 44 et 45 .
} 
- d'un autre côté, le système de mise en vigueur précédé de missions d'évaluation a eu pour résultat que les règles des accords de Schengen furent appliquées effectivement en pratique.

\subsection{Au sujet de la coopération renforcée}

Le principe de la coopération renforcée a longtemps été contesté. L'idée d'une Europe à plusieurs vitesses, ou d'une Europe à géométrie variable était toutefois avancée de tous les côtés. ${ }^{56} \mathrm{Au}$ fur et à mesure que l'Europe s'élargissait, il paraissait évident qu'une certaine différenciation devenait inévitable. Pour Jacques Delors ${ }^{57}$ la différenciation constitue la seule solution possible pour concilier l'élargissement et l'approfondissement. Pour d'autres, la coopération renforcée mène au morcellement du droit européen. ${ }^{58}$ De fait, la coopération renforcée a été introduite par le Traité d'Amsterdam (TUE, Titre VII, articles 43 à 45), sous des conditions par ailleurs très strictes. 59

Force est de reconnaître, qu'elle n'a longtemps pas eu de succès. Les accords de Schengen constituent une coopération renforcée en dehors des traités et n'ont été incorporés dans les traités que près de dix ans après leur conclusion et près de cinq ans après leur mise en vigueur. ${ }^{60}$

La coopération, connue sous le nom de Traité de Prüm, est également une coopération renforcée en dehors des traités. Poursuivant l'objectif de renforcement de la coopération policière par l'amélioration des échanges d'informations, par l'échange d'empreintes digitales et la constitution de patrouilles policières communes, l'instrument signé à Prüm le 27 mai 2005 par sept États membres a très rapidement été repris presque intégralement dans l'ordre juridique de l'Union européenne par l'ensemble des États membres. ${ }^{61}$

Dans les deux cas - Schengen et Prüm - on constate la force de l'effet d'entrainement qui amène le ralliement de beaucoup d'États hésitants au moment du lancement de l'initiative. Dans le cas du Traité de Prüm, ce mouvement a été particulièrement rapide. ${ }^{62}$ Il n'en reste pas moins que dix ans après l'entrée en vigueur du Traité d'Amsterdam aucune coopération renforcée n'avait été tentée, ni réussie, dans le cadre des traités. Finalement la première coopération renforcée sera lancée dans le domaine de

\footnotetext{
${ }^{56}$ Voir Dennewald [4], p. 170 et de Kerchove [1], p. 17 à 20.

${ }^{57}$ Delors [3], p. 463.

${ }^{58}$ Brigitte Zypries, ministre allemande de la Justice, citée dans Dennewald [4], p. 188.

${ }^{59}$ Sur cette question et le développement ultérieur dans les traités de Nice et de Lisbonne, voir Dennewald [4], p. 178 et suivantes.

${ }^{60}$ Avec le traité d'Amsterdam ils acquièrent le statut de première coopération renforcée puisque l'article $1^{\text {er }}$ du Protocole Schengen stipule que les 13 États membres de l'Union qui sont «signataires des accords de Schengen sont autorisés à instaurer entre eux une coopération renforcée dans les domaines relevant du champ d'application desdits accords. »

${ }^{61}$ Décision 2008/615 JAI relative à l'approfondissement de la coopération transfrontalière, notamment en vue de lutter contre le terrorisme et la criminalité transfrontalière (JO L 201 du 6.8.2008).

${ }^{62}$ Sur les questions relatives au Traité de Prüm voir séminiaire ERA du 24-25.5.2007 «Der Vertrag von Prüm » et notamment la contribution de M. Niemeier.
} 
la coopération judiciaire civile. Après des hésitations initiales de la Commission Barroso I - estimant que les États membres désireux de prendre le chemin d'une coopération renforcée dans la question des divorces transfrontaliers ne constituaient pas une «masse critique suffisante pour aller de l'avant»-Viviane Reding, vice-présidente de la nouvelle Commission Barroso entrée en fonction début 2010 présentait rapidement une initiative. ${ }^{63}$ Actuellement, et sur base d'une décision du Conseil autorisant une coopération renforcée dans le domaine du droit applicable au divorce et à la séparation de corps entre neuf États membres les instances du Conseil viennent de finaliser la première coopération renforcée en matière de coopération judiciaire civile. ${ }^{64}$

Encore une fois, l'effet d'entrainement a finalement joué et d'autres États membres vont suivre le mouvement. On serait donc tenté de conclure - après ces différents exemples relevant tant des aspects de liberté (Schengen), de sécurité (Schengen et Prüm) et de justice (divorce transnational) - que l'ensemble de l'espace de liberté, de sécurité et de justice peut désormais progresser grâce aux possibilités offertes par la coopération renforcée. Il est vrai néanmoins, que les coopérations renforcées hors traité seront désormais plus difficiles en raison des dispositions de l'article 218 TUE réglant les compétences externes de la Communauté. ${ }^{65}$

\section{Conclusions}

On peut considérer que l'initiative Schengen a été une chance pour l'Europe. Développée à partir d'une action menée par cinq États membres fondateurs des Communautés Européennes, elle s'est développée en dehors des structures des traités tout en se subordonnant aux progrès à réaliser dans les instances des Communautés, puis de l'Union européenne.

Intégrés dans une deuxième phase dans les traités européens, les accords de Schengen ont été pour ceux-ci un élément de progrès : il est permis d'affirmer que les problèmes relatifs à l'abolition des contrôles aux frontières intérieures n'auraient pas trouvé de solution satisfaisante au sein de l'Europe des dix, des douze ou des quinze ; de même, les progrès dans des domaines essentiels comme ceux de la coopération policière n'auraient guère pu être réalisés dans de tels délais. ${ }^{66}$

Ainsi Schengen apparaît, en définitive, comme un accélérateur et un facilitateur de l'espace de liberté, de sécurité et de justice. Le volumineux acquis de Schengen, fruit d'une coopération d'une quinzaine d'années dans un cadre informel, constitue un apport substantiel à la construction européenne et une des grandes avancées de l'Europe.

\footnotetext{
${ }^{63}$ Voir Dennewald [4], p. 188.

${ }^{64}$ Règlement du Conseil adopté à 14 fin décembre 2010.

${ }^{65}$ Voir Dennewald [4], p. 191 qui examine quelques pistes pour préserver les possibilités d'une coopération intergouvernementale.

${ }^{66}$ L'Union européenne le reconnaît elle-même indirectement dans le premier considérant du Protocole Schengen annexé au Traité d'Amsterdam qui note que «les accords (de Schengen...) visent à renforcer l'intégration européenne et, en particulier, à permettre à l'Union européenne de devenir plus rapidement un espace de liberté, de sécurité et de justice » (c'est nous qui soulignons).
} 
Mais au-delà des analyses sur les mérites des différents instruments juridiques dans la construction européenne, c'est peut-être sur l'opinion du citoyen européen qu'il convient de conclure. Assurément ce droit de libre circulation acquis grâce aux accords de Schengen constitue - ensemble avec l'euro - l'exemple le plus concret, vécu au jour le jour, par le citoyen européen. Cela est plus visible encore pour les citoyens de l'Europe de l'Est pour lesquels, autrefois, les frontières étaient difficilement franchissables. L'adhésion de ces pays en $2004^{67}$ ne signifiait pas immédiatement et automatiquement participation à l'espace Schengen et ne signifiait donc pas libre circulation des personnes ni ouverture des frontières. Des aménagements rendus nécessaires pour faire fonctionner le SIS (Système Informatique Schengen) ayant retardé cette accession, ce ne fut finalement que le 21 décembre 2007 que l'espace Schengen fut étendu à ces États. Dans une séance académique organisée dans

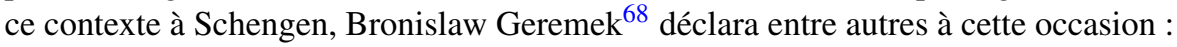
«La suppression des frontières internes de l'UE, c'est la marque de reconnaissance que tous les citoyens des États concernés appartiennent au même espace, et qu'ils partagent une identité commune ».

La même idée, décrivant le sentiment des citoyens des nouveaux États membres, a été résumée en une formule saisissante par Jacienka Wilczac : «La véritable Europe, c'est celle de Schengen $\gg .{ }^{69}$

\section{Bibliographie}

1. de Kerchove, G.: In: Delpérée, F. (ed.) Mélanges. Bruylant, Brussels (2007)

2. de Lobkowicz, W.: L'Europe et la sécurité intérieure, La Documentation Française (2002)

3. Delors, J.: Mémoires. Plon, Paris (2004)

4. Dennewald, J.: L'espace judiciaire européen au lendemain du Traité de Lisbonne : état des lieux et perspectives de l'intégration différenciée. In: ERA Forum 2/2010. Springer, Berlin (2010)

5. Elsen, C.: Les structures administratives de Schengen. In: Pauly (ed.) Les accords de Schengen : Abolition des frontières intérieures ou menace pour les libertés publiques. EIPA, Maastricht (1993)

6. Elsen, C.: Incorporation juridique et institutionnelle de Schengen dans l'UE. In: Den Boer, M. (ed.) Schengen Still Going Strong. EIPA, Maastricht (2000)

7. Fally, V.: Le Grand-Duché de Luxembourg et la Construction européenne. Saint-Paul, Luxembourg (1992)

8. Fischbach, M.: La politique de Schengen. In: Pauly (ed.) Les accords de Schengen. EIPA, Maastricht (1993)

9. Papagianni, G.: Institutional and Policy Dynamics of EU Migration Law. Nijhoff, Dordrecht (2006)

\footnotetext{
${ }^{67}$ Estonie, Hongrie, Lettonie, Lithuanie, Pologne, Slovaquie, Slovénie, République Tchèque.

${ }^{68}$ Ancien Ministre des Affaires Etrangères de Pologne, ancien parlementaire européen.

${ }^{69}$ Polityka Varsovie 20.4.2010.
} 